\title{
PERFIL SOCIAL DE USUÁRIOS E DO SERVIÇO DE FARMÁCIA DO CENTRO DE ATENÇÃO ESPECIALIZADA DO IDOSO EM BELÉM, PARÁ
}

\author{
Ingrid Wini da Silva Pereira ${ }^{1}$, Francisco Matias Dias ${ }^{1}$, Adriellen Saraí de Lima Gonçalves ${ }^{1}$, \\ Orenzio Soler ${ }^{1}$ \\ ${ }^{1}$ Universidade Federal do Pará \\ E-mail para correspondência: orenziosoler@gmail.com \\ Submetido em: 28/08/2020 e aprovado em: 18/12/2020
}

\begin{abstract}
RESUMO
Introdução: Este artigo trata de indicadores relacionados a determinantes sociais, equidade, política de saúde da pessoa idosa, perfil de prescrições e do atendimento farmacêutico, critérios de Beers-Fick, interações de medicamentos e grau de conhecimento e satisfação de utentes, possibilitando inferir um juízo ágil e baseado em critérios técnicos. Objetivo: Investigar o perfil social de usuários e do serviço de farmácia do Centro de Atenção Especializada do Idoso em Belém, Pará. Metodologia: Trata-se de um estudo transversal, exploratório, retrospectivo, qualitativo/quantitativo, utilizando-se de amostra por conveniência de 100 idosos ( $\geq 65$ anos) de ambos os sexos. Resultados: 36\% possuem cadastro na Estratégia Saúde da Família. 50,0\% relataram ter assistência social. 53\% relataram consumir cinco ou mais porções diárias de alimentos, incluindo frutas, legumes e verduras. $0,0 \%$ relatou ingerir altas doses de álcool. 2\% relataram ser fumantes. $45 \%$ relataram ser fisicamente inativos. 97,0\% relataram residir em domicílios adequados. 57\% relataram ter amigos na vizinhança e participar de grupos da igreja. $13,0 \%$ relataram não ter limitações. $0,0 \%$ não relatou violência e internação de idosos por maus-tratos. $13,8 \%$ dos medicamentos padronizados se enquadram nos Critérios de Beers-Fick. $16 \%$ das prescrições apresentaram potenciais interações de medicamentos classificadas como sérias. Os achados quanto ao perfil de dispensação de medicamentos permitem inferir que a estratégia do cuidado farmacêutico ainda não foi implantado. 98\% auto-referiram se sentir respeitado, 99\% se senti bem e 97\% gostam de frequentar o Centro de Atenção Especializado ao Idoso. Conclusão: Os achados relacionados a indicadores de determinantes sociais, equidade e política de saúde da pessoa idosa permitem inferir que os usuários do Centro de Atenção Especializado ao Idoso seguem o perfil nacional. Os indicadores de perfil de prescrições, critérios de Beers-Fick, interações de medicamentos, dispensação farmacêutica permitem inferir a necessidade do aprimoramento do serviço farmacêutico. Por fim, quanto ao conhecimento dos utentes sobre a sua farmacoterapia e sua satisfação com o atendimento foram elevados.
\end{abstract}

Palavras-chave: Políticas Públicas. Política do Idoso. Saúde do Idoso. Assistência Farmacêutica. Serviço Farmacêutico.

\section{SUMMARY}

Introduction: This article deals with indicators related to social determinants, equity, health policy for the elderly, prescriptions and pharmaceutical care profile, Beers-Fick criteria, drug interactions and degree of knowledge and user satisfaction, making it possible to infer a agile judgment based on technical criteria. Objective: To investigate the epidemiological profile of users and the pharmacy service of the Specialized Care Center for the Elderly in Belém, Pará. 
Methodology: This is a cross-sectional, exploratory, retrospective, qualitative / quantitative study, using a sample for convenience. 100 elderly ( $\geq 65$ years) of both sexes. Results: $36 \%$ are registered in the Family Health Strategy. 50.0\% reported having social assistance. 53\% reported consuming five or more servings of food daily, including fruits and vegetables. $0.0 \%$ reported drinking high doses of alcohol. $2 \%$ reported being smokers. $45 \%$ reported being physically inactive. $97.0 \%$ reported living in adequate households. 57\% reported having friends in the neighborhood and participating in church groups. $13.0 \%$ reported not having limitations. $0.0 \%$ did not report violence and hospitalization of the elderly for mistreatment. $13.8 \%$ of standardized drugs meet the Beers-Fick criteria. $16 \%$ of prescriptions had potential drug interactions classified as serious. The findings regarding the medication dispensing profile allow us to infer that the pharmaceutical care strategy has not yet been implemented. $98 \%$ selfreported feeling respected, 99\% felt good and 97\% like to attend the Specialized Care Center for the Elderly. Conclusion: The findings related to indicators of social determinants, equity and health policy for the elderly allow us to infer that users of the Specialized Care Center for the Elderly follow the national profile. The prescription profile indicators, Beers-Fick criteria, drug interactions, pharmaceutical dispensation allow us to infer the need to improve the pharmaceutical service. Finally, the users' knowledge about their pharmacotherapy and their satisfaction with the service were high.

Key words: Public policy. Elderly Policy. Health of the Elderly. Pharmaceutical care. Pharmaceutical Service.

\section{INTRODUÇÃO}

A população brasileira ao longo dos anos vem sofrendo uma transição demográfica acelerada na sua pirâmide etária, em que observamos um rápido envelhecimento. Assim, sendo, tem-se diretrizes e políticas públicas para garantir o envelhecimento ativo com segurança e qualidade de vida ${ }^{(1)}$.

A problemática dos países em desenvolvimento é prestar assistência à crescente população de idosos - que majoritariamente -, é composta por pessoas com baixo nível socioeconômico e educacional; assim como, da prevalência de doenças crônicas que demandam cuidado permanente para evitar incapacidades e deficiências ${ }^{(2)}$.

Os instrumentos de proteção à pessoa idosa no ordenamento jurídico brasileiro iniciam com a Constituição Federal de 1988, tendo prosseguimento com a Política Nacional do Idoso (PNI) em 1994, o Estatuto do Idoso em 2003, a Política Nacional de Saúde da Pessoa Idosa (PNSPI) em 2006; bem como, de acordos internacionais como o Plano de Ação Internacional para o Envelhecimento de $2003^{(3)}$. Entretanto, a falta de indicadores para acompanhamento dessas políticas é um obstáculo para sua consolidação.

\section{Política Nacional de Saúde da Pessoa Idosa}

A Lei $n^{\circ} 8.080$, de 19 de setembro de 1990, dispõe sobre as condições para a promoção, 
proteção e recuperação da saúde, a organização e o funcionamento dos serviços de saúde correspondentes e da outras providências ${ }^{(4)}$. A mesma estabelece que a saúde é um direito de todo e qualquer cidadão e que o Estado é responsável por prover condições indispensáveis ao seu pleno exercício.

A Política Nacional do Idoso, regida pela Lei $n^{\circ} 8.842$, de 4 de janeiro de $1998,{ }^{(5)}$ tem por objetivo assegurar os direitos sociais do idoso, criando condições para promover sua autonomia, integração e participação efetiva na sociedade, dispondo de princípios, que assegura que a, família, sociedade e estado sejam responsáveis por garantir a cidadania, garantindo sua participação na comunidade; bem como, defendo sua dignidade e direito à vida; assim como, de diretrizes que viabilizam formas alternativas de participação, ocupação e convívio do idoso em sua geração e gerações futuras.

O Estado tem por dever garantir a saúde, buscando formular e reformular a execução de políticas econômicas e sociais, a diminuição de riscos de doenças e de agravos, visando assegurar acesso universal e equitativo às ações e serviços para a promoção, proteção e recuperação a saúde. Desse modo, a família, a comunidade, a sociedade e o poder público tem por obrigação assegurar ao idoso o direito à vida, à saúde, educação, alimentação, cultura, esporte, lazer, trabalho, cidadania, liberdade, dignidade, respeito e à convivência familiar e comunitária $^{(6,7)}$.

O Estatuto do Idoso, amparado pela Lei de $\mathrm{n}^{\circ} 10.741$, de 1 de outubro de 2003, destina recursos para regular os direitos assegurados para as pessoas com idade igual ou superior a 60 (sessenta) $\operatorname{anos}^{(6)}$. O idoso tem por direito usufruir de todos os direitos fundamentais relacionados a pessoa humana, o qual desfruta de proteção integral; sendo esse, assegurado por lei e por outros meios, tendo oportunidade e facilidade para que sua saúde física e mental sejam preservadas.

A Política Nacional de Saúde da Pessoa Idosa, normatizada pela Portaria MS/GM n ${ }^{\circ}$ 2.528, de 20 de outubro de 2006, ${ }^{(7)}$ estabelece medidas conjuntas e individualizadas de saúde para a população idosa de modo articulado com os princípios e diretrizes do Sistema Único de Saúde (SUS), visando estimular a autonomia e independência da pessoa idosa.

\section{Assistência à saúde a pessoa idosa}

O processo de envelhecer é direito de todos, amparado por lei a qual é responsável por garantir que a pessoa idosa passe ilesa e gozando dos plenos benefícios oferecidos; bem como, a saúde é direito de todos e dever do Estado ${ }^{(8,9)}$. Uma das maiores conquistas da humanidade 
foi a longevidade. Tal conquista só foi possível devido à grande melhora que se obteve em parâmetros de saúde; mesmo que, não contemple de forma igualitária a todos. Conquistar a velhice, antes era privilégio, hoje passou a ser normal mesmo com todos os desafios.

A transição demográfica e a melhoria dos indicadores sociais e econômicos do Brasil em comparação com décadas anteriores -, trouxeram a ampliação do contingente de idosos e maior pressão fiscal sobre os sistemas de saúde público e privado. Desse modo, se teve o aumento generoso da parcela de idosos e, consequentemente, o aumento de doenças crônicas e gastos, demandando serviços de saúde complexos, redobrando as internações hospitalares; bem como, a frequência e tempo que os leitos são ocupados ${ }^{(8,9)}$.

Os programas voltados ao público idoso devem ser construídos com base na integralidade do cuidado com protagonismo profissional, senso de preferência de uma equipe multidisciplinar de saúde; gerenciando não a doença, mas o perfil de saúde do utente. Sabe-se que a doença crônica apresenta características permanentes, produzindo incapacidades, cursando lentamente e apresentando causas diversas. Porém, podem ser corrigidos com mudanças nos hábitos de vida e cuidados que perduraram a vida toda, mesmo que não levem a cura, mas que permitam o controle das morbidades e o prolongando do tempo de vida com qualidade $^{(6,7)}$.

Em adição, as morbidades que acometem os idosos são de caráter crônico e múltiplas, exigindo acompanhamento constante em diversas especialidades. Por possuir tanta adversidade, os idosos precisam de um sistema de saúde estruturado, garantindo cuidados redobrados. Entretanto, ainda temos problemas em função de um sistema de saúde fragmentado; ou seja, organizado por especialidades, não permitindo o cuidado integral ao idoso. Este modelo de saúde gera mais gastos; caracterizando-se por ineficiente ${ }^{(8,9)}$.

\section{Assistência farmacêutica à pessoa idosa}

Há evidências robustas da necessidade de um olhar e um cuidado diferenciado para a saúde do idoso. Os idosos tem uma particularidade; ou seja, em sua maioria são portadores de doenças crônicas. Neste contexto, é comum utilizarem distintas estratégias farmacológicas e não farmacológicas. Nos países desenvolvidos os profissionais vem sendo impulsionados a desenvolverem ferramentas para identificar padrões de prescrições inadequadas e problemas farmacoterapêuticos ${ }^{(10,12)}$.

Assim, sendo, Beers e Fick, vem apresentando listas de medicamentos potencialmente inapropriados para idosos, seguindo uma abordagem baseada em evidências. A última revisão 
da Lista de Beers-Fick foi feita em 2015(12). Há medicamentos inapropriados devido as interações doença-medicamento e/ou síndrome-medicamento. Os medicamentos que devem ser usados com precaução. As interações medicamentosas clinicamente importantes que devem ser evitadas. Os medicamentos que devem ser evitados ou ter a posologia reduzida de acordo com os níveis de função renal em idosos e os que possuem fortes propriedades anticolinérgicas.

A Lista de Beers-Fick se apresenta como um instrumento importante para a realização do cuidado farmacêutico, auxiliando os profissionais prescritores e dispensadores a evitarem erros de medicação; em especial, a prescrição de medicamentos inapropriados para os idosos em polifarmácia, o que acarreta custos humanos, econômicos e sociais ${ }^{(12,13)}$.

A polifarmácia ou polimedicação é conceituada como o uso de vários medicamentos de forma concomitante; sendo que, muitos autores usam o termo para o uso de distintos medicamentos dentro de um mesmo período de tempo ${ }^{(3,8)}$. A polifarmácia está associada a prescrição de múltiplos medicamentos para um indivíduo. Sabe-se que os idosos passam por mudanças orgânicas significativas; em especial, aquelas relacionadas a alterações fisiológicas como farmacocinéticas e farmacodinâmicas ${ }^{(13)}$. A utilização de muitos medicamentos potencialmente inapropriados pode gerar eventos de hospitalização e alto custo ao sistema de saúde, com isso a ineficiência terapêutica.

Por fim, reconhece-se conquistas importantes para os idosos; em especial, a Lei 10.741 de 1 de outubro de 2003, que estabeleceu o Estatuto do Idoso; bem como, a Portaria MS/GM n 2.528 de 19 de outubro de 2006 que implementou a Política Nacional de Saúde do Idoso, instrumentos considerados indispensáveis à inclusão funcional de idosos e à promoção do envelhecimento com menores riscos de agravos à saúde ${ }^{(10,11)}$. Neste contexto, este estudo teve por objetivo investigar o perfil social de usuários e do serviço de farmácia do Centro de Atenção Especializada do Idoso em Belém, Pará.

\section{MÉTODOS}

Trata-se de um estudo transversal, retrospectivo, de natureza qualitativa/quantitativa, ${ }^{(14,15)}$ realizado em 2019, utilizando-se de amostragem por conveniência, tendo como critérios de inclusão ser idoso com 65 anos ou mais, ter polimorbidades, ser de alto risco, de ambos os sexos e utentes assíduos ao Centro de Atenção Especializado ao Idoso (Casa do Idoso), vinculado à Secretaria Municipal de Saúde da Prefeitura Municipal de Belém (SESMA/PMB).

O Centro de Atenção Especializado ao Idoso (CNES nº 7598505), tem 9.536 idosos 
cadastrados. Conta com um corpo técnico de 5 clínicos; 1 gastroenterologista; 2 urologistas; 3 geriatras; 1 endocrinologista; 1 ginecologista; 1 ortopedista; 3 odontólogos; 3 enfermeiros; 2 nutricionistas; 1 psicólogo; 1 fonoaudiólogo; 3 terapeutas ocupacionais; 3 assistentes sociais e 3 farmacêuticos. Atende pessoas acima de 60 anos encaminhadas por unidades básicas de saúde do município, disponibilizando clínica médica, geriatria, dermatologia, cardiologia, reumatologia, enfermagem, fisioterapia, terapia ocupacional, odontologia, fonoaudiologia, psicologia, assistência farmacêutica; assim, como, oficinas de educação em saúde.

Sabe-se que indicadores são utilizados como forma de avaliação e monitoramento de serviços, permitindo realizar comparações das condições dos serviços prestados e do nível de resolutividade das ações desenvolvidas. É relevante empregá-los em políticas públicas, pois fornecem dados para o planejamento das intervenções, para monitorar a implementação de programas e avaliar seus resultados.

Para investigar sobre os determinantes sociais e de equidade utilizou-se o PROGRESS ( $\mathrm{P}=$ Local de residência; $\mathrm{R}=$ Raça/etnia/cultura/idioma; $\mathrm{O}=$ Ocupação; $\mathrm{G}=$ Sexo/gênero; $\mathrm{R}=$

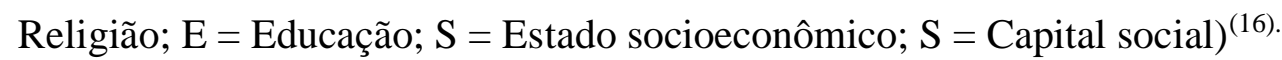

Utilizou-se do método preconizado por Silveira et al., ${ }^{(3)}$ para se conhecer o perfil social dos utentes, fundamentados em indicadores para distintas dimensões da saúde: Acesso aos serviços de saúde; Prevenção e manutenção da capacidade funcional; Violência e maus-tratos; Moradia adequada; Participação na comunidade; Assistência social. Em adição, para fazer comparação, buscou-se dados e informações no Sistema de Informações em Saúde (SIS) do Ministério da Saúde, nos censos demográficos do Instituto Brasileiro de Geografia e Estatística de 2000 e 2010, no Suplemento Saúde da Pesquisa Nacional por Amostra de Domicílio (Pnad) de 2008, na Pesquisa Nacional de Saúde (PNS) de 2013 e nos dados administrativos do Ministério de Desenvolvimento Social (MDS).

Para se obter dados e informações relacionados aos aspectos técnico-assistenciais do serviço de farmácia, seguiu-se os caminhos de Pereira, ${ }^{(17)}$ e Likert, ${ }^{(18)}$; ou seja, entrevistas semiestruturadas com profissionais e utentes quanto ao perfil das prescrições, potenciais interações medicamentosas, perfil de dispensação farmacêutica, grau de conhecimento sobre a farmacoterapia e satisfação do utente quanto aos serviços prestados.

Para a avaliação das prescrições, seguiu-se o modelo do fluxograma do tipo top-douw ${ }^{(19)}$ observando se a receita contemplava todos os critérios: Características do utente: nome completo, idade, sexo e peso; Características do prescritor: nome completo, especialidade, número do registro em conselho e assinatura e carimbo; Características do medicamento: nome 
genérico, concentração/dosagem, forma farmacêutica, quantidade total necessária; Instruções de uso: via de administração, dose unitária, intervalo, duração de tratamento, observações especiais e data.

Acredita-se que com estes indicadores é possível realizar um juízo ágil e baseado em critérios técnicos sobre o estado geral de uma determinado campo temático, apesar de não serem suficientes para fazer um julgamento acurado sobre o contexto de um programa ou política. Os dados foram plotados no Microsoft Office Excel ${ }^{\circledR}$ (2016) e tratados para serem apresentados em frequências absoluta e relativa e por meio de síntese narrativa. Foram cumpridos os requisitos éticos, sendo o projeto submetido à Plataforma Brasil sob o CAAE $n^{\circ}$ 15259119.0.0000.0018 e aprovado via Protocolo $\mathrm{n}^{\mathrm{o}} 3.579 .922$.

\section{RESULTADOS E DISCUSSÃO}

Foram analisados 100 prontuários de idosos ambos os gêneros, com 65 anos ou mais, com polimorbidades e de alto risco. Verificou-se que $77,3 \%(n=75)$ são do sexo feminino e $22,7 \%(\mathrm{n}=25)$ do sexo masculino, sendo a faixa de idade entre 65 a 99 anos, média de idade \pm desvio padrão de 79,6 $\pm 6,0 ; 80,2 \pm 5,9$ anos para mulheres e $77,5 \pm 5,8$ para homens.

\section{Indicadores de determinantes sociais, equidade e da política de saúde da pessoa idosa de usuários do Centro de Atenção Especializada do Idoso}

Quanto ao perfil sociodemográfico, epidemiológico, econômico e de equidade, observou-se um predomínio de residência urbana $(100 \%)(\mathrm{n}=100)$, raça parda $(73 \%)(\mathrm{n}=73)$, aposentados $(50 \%)(n=50)$, católicos $(73 \%)(n=73)$, ensino fundamental $(79 \%)(n=79)$, renda familiar de 1 a $3(79 \%)(n=79)$, capital social $(70 \%)(n=70)$ e de 4 a 7 habitantes por domicílio $(56 \%)(\mathrm{n}=56)($ Tabela 1), sendo os achados são semelhantes aos perfis encontrados por outros autores $^{(17,24-30)}$.

Tabela 1 - Indicadores de determinantes sociais, equidade e da política de saúde da pessoa idosa

\begin{tabular}{|c|c|c|c|c|c|c|c|c|}
\hline \multirow{2}{*}{ AMOSTRA } & \multicolumn{8}{|c|}{ DETERMINANTES SOCIAIS E DE EQUIDADE* } \\
\hline & $\mathrm{P}(\%)$ & $\mathrm{R}(\%)$ & $\mathrm{O}(\%)$ & $\mathrm{G}(\%)$ & $\mathrm{R}(\%)$ & $\begin{array}{c}\mathrm{E} \\
(\%)\end{array}$ & $\mathrm{S}(\%)$ & $\mathrm{S}(\%)$ \\
\hline Mulheres $(n=75)$ & 100 & 74,0 & 84,0 & 77,3 & 13,3 & 72,0 & 32,0 & 78,6 \\
\hline Homens $(n=23)$ & 100 & 72,7 & 77,2 & 22,7 & 4,5 & 63,3 & 31,8 & 77,2 \\
\hline \multicolumn{9}{|c|}{ POLÍTICA DA PESSOA IDOSA** } \\
\hline \multicolumn{4}{|c|}{ Área Temática } & \multicolumn{2}{|c|}{ Brasil*** } & \multicolumn{3}{|c|}{ Casa do Idoso } \\
\hline \multicolumn{4}{|c|}{ Acesso aos serviços de saúde: ESF } & \multicolumn{2}{|c|}{$5,4 \%$} & \multicolumn{3}{|c|}{$36,0 \%$} \\
\hline \multicolumn{4}{|l|}{ Assistência social } & \multicolumn{2}{|c|}{$6,95 \%$} & \multicolumn{3}{|c|}{$50,0 \%$} \\
\hline
\end{tabular}




\begin{tabular}{|c|c|c|}
\hline Estilo de vida: Alimentação adequada & $40,1 \%$ & $53,0 \%$ \\
\hline Estilo de vida: Consumo abusivo e frequente de álcool em idosos & $14,2 \%$ & $0,0 \%$ \\
\hline Estilo de vida: Idosos fisicamente inativos & $62,7 \%$ & $45,0 \%$ \\
\hline Estilo de vida: Proporção de idosos fumantes & $13,3 \%$ & $2,0 \%$ \\
\hline Moradia adequada & $69,3 \%$ & $97,0 \%$ \\
\hline Participação social & 46,3 & $57,0 \%$ \\
\hline Prevenção e manutenção da capacidade funcional & $14,29 \%$ & $13,0 \%$ \\
\hline Violência e maus-tratos & $0 \%$ & $0 \%$ \\
\hline \multicolumn{3}{|c|}{$\begin{array}{c}\text { Legenda: } \mathbf{P}=\text { Local de residência } ; \mathbf{R}=\text { Raça/etnia/cultura/idioma; } \mathbf{O}=\text { Ocupação; } \mathbf{G}=\text { Sexo/gênero; } \mathbf{R}=\text { Religião; } \mathbf{E}= \\
\text { Educação; } \mathbf{S}=\text { Estado socioeconômico; } \mathbf{S}=\text { Capital social. }\end{array}$} \\
\hline \multicolumn{3}{|c|}{ * Adaptado de: O'neill et al. (2014). } \\
\hline \multicolumn{3}{|c|}{$\begin{array}{c}\text { ** Adaptado de: Silveira et al. }{ }^{3} \text { Amostra: } 100 \text { utentes. Não foram encontrados dados para o estado do Pará e município de } \\
\text { Belém. }\end{array}$} \\
\hline
\end{tabular}

Fonte: Indicadores de saúde e de assistência farmacêutica voltadas à população idosa no Centro de Atenção Especializada do idoso de Belém, 2020.

Quanto ao acesso aos serviços de saúde, 36\% $(\mathrm{n}=36)$ dos idosos usuários do CAEI possuíam cadastro na Estratégia Saúde da Família $(E S F) .62 \%(n=62)$ relataram que utilizam apenas o CAEI, devido à falta de médicos e morosidade dos serviços nas unidades da Atenção Primária à Saúde (APS) (Tabela 1). No que tange ao acesso aos serviços de saúde, os dados da Pnad e da PNS mostram que, de maneira geral, o acesso aos serviços básicos de saúde melhorou no Brasil entre 2008 e 2013, passando de 48,6\% dos idosos cobertos para 56,3\% neste período. A adesão à ESF foi maior no Nordeste e no Sul do país, mas a maior expansão do programa no período considerado aconteceu no Sudeste (de 35,8\% para 48,7\%). Vale destacar o caso do Norte, única região cuja cobertura da ESF para a população idosa permaneceu estável no período $^{(3)}$.

Quanto a assistência social, 50,0\% $(\mathrm{n}=50)$ dos idosos usuários do CAEI relataram ter assistência social. Pesquisadores que estimaram o número potencial de pessoas idosas elegíveis ao Benefício de Prestação Continuada (BPC), utilizando dados do Censo 2000 e compararam com o número de beneficiários nos registros administrativos, em 2005, para avaliar cobertura, concluíram que, em 2005, o benefício atingia 100\% de seu público-alvo, com uma leve sobrecobertura para homens $(115 \%)$ e subcobertura para mulheres $(97 \%)^{(3)}$.

Quanto a alimentação adequada, 53\% $(\mathrm{n}=53)$ dos idosos usuários do CAEI relataram consumir cinco ou mais porções diárias de alimentos, incluindo frutas, legumes e verduras (Tabela 1). Sobre o estilo de vida das pessoas idosas, os dados da Pesquisa Nacional de Saúde 
(PNS) nos permitem observar que, em 2013, apenas 32,2\% dos idosos brasileiros se alimentavam adequadamente. A região com os piores resultados foi o Nordeste $(25,1 \%)$ e a que obteve os melhores foi o Centro-Oeste $(39,5 \%)^{(3)}$.

Quanto ao consumo abusivo e frequente de álcool, 0,0\% $(\mathrm{n}=0)$ dos idosos usuários do CAEI relatou ingerir altas doses de álcool (oito ou mais doses para mulheres e 15 ou mais doses para homens); apenas consumindo esporadicamente em ocasiões especiais (Tabela 1). Dados da Pesquisa Nacional de Saúde (PNS) nos permitem observar que, em 2013, 24,1\% dos idosos brasileiros consomem álcool de forma abusiva e frequente, mas existe uma variação intensa entre as regiões; com algumas, como o Norte, tendo uma proporção muito menor do que a média nacional $(14,8 \%)$ e outras, como o Sul, muito superior $(30,3 \%)^{(3)}$.

Quanto a fumar, 2\% $(\mathrm{n}=2)$ dos idosos usuários do CAEI relataram ser fumantes (Tabela 1). Para a proporção de idosos fumantes temos dados que permitem a comparação entre dois pontos no tempo. É possível observar que, entre 2008 e 2013, o percentual caiu no Brasil cerca de $1,85 \%$. Apesar de todas as regiões terem registrado queda, em algumas, como o Norte (3,85\% de queda), Centro-Oeste $(3,5 \%)$ e Nordeste $(3,1 \%)$, essa diminuição foi muito maior que em outras regiões, como o Sudeste $(0,99 \%)$ e o Sul $(0,47 \%)^{(3)}$.

Quanto a ser fisicamente inativos, 45\% $(\mathrm{n}=45)$ dos idosos usuários do CAEI relataram ser fisicamente inativos (Tabela 1). Quanto à inatividade física entre pessoas idosas, em razão da mudança da pergunta, entre a Suplemento Saúde da Pesquisa Nacional por Amostra de Domicílio (Pnad) 2008 e a Pesquisa Nacional de Saúde (PNS) 2013 não é possível fazer uma série histórica. Todas as regiões têm, em média, 30\% de pessoas idosas inativas fisicamente, sendo o percentual ligeiramente maior na região Sul (45,7\%). Quanto à participação social, $25 \%$ das pessoas idosas frequentam atividades sociais organizadas de forma frequente. Esse número é maior no Sul $(30,2 \%)$ e menor no Nordeste $(22,1 \%)^{(3)}$.

Quanto a viver em domicílios adequados, 97,0\% $(\mathrm{n}=97)$ dos idosos usuários do CAEI relataram residir em domicílios adequados (Tabela 1). A moradia adequada - com esgotamento sanitário, coleta de lixo e água encanada - é um dos fatores ambientais mais importantes para a saúde da população. O Brasil tem avançado neste aspecto, passando de 60,1\%, em 2000, para 69,3\% em 2010. A região com maior proporção de idosos em moradia adequada é o Sudeste, cerca de 78,69\% em 2010; contudo, é notável o avanço de outras regiões neste sentido. Dentre elas, podemos destacar o Centro-Oeste, que teve um aumento de 11,63\% em 10 anos, passando de 60,84\%, em 2000, para 72,47\% em $2010^{(3)}$.

Quanto a participação social, 57\% $(\mathrm{n}=57)$ dos idosos usuários do CAEI relataram ter 
amigos na vizinhança e participar de grupos da igreja próximo ao domicílio, devido a facilidade de deslocamento (Tabela 1). A trajetória de participação social mais comumente descrita na literatura é de redução das redes sociais e diminuição da participação em atividades sociais; ou seja, de desengajamento social, tanto com valor negativo, quanto como processo de seleção intencional e adaptativa. As teorias mais presentes na literatura foram a teoria da atividade (de forma implícita) e a teoria da seletividade socioemocional (de forma explícita). Esses resultados apontam para a necessidade de reflexão mais cuidadosa sobre o que é idealizado e o que é praticado em termos de políticas e práticas para alcançar o envelhecimento bem sucedido. Os esforços futuros devem contemplar não apenas o incentivo à atividade, mas os aspectos que influenciam o desengajamento social associado à má adaptação dos idosos ${ }^{(20)}$.

Quanto a limitação funcional para realizar atividades de vida diária (AVD), 13,0\% ( $\mathrm{n}=$ 13) dos idosos usuários do CAEI relataram não ter limitações (Tabela 1). Quanto à prevenção e manutenção da capacidade funcional, vemos que 6,8\% dos idosos brasileiros têm alguma limitação funcional para realizar as AVD. As regiões com mais pessoas idosas nesta situação são o Nordeste $(8,3 \%)$ e o Centro-Oeste $(8,1 \%)$, e a que tem menos é o Norte $(5,7 \%)^{(3)}$.

Quanto a violência e internação de idosos por maus-tratos, $0,0 \% \quad(n=0)$ dos idosos usuários do CAEI não relatou violência e internação de idosos por maus-tratos (Tabela 1). Sobre a área temática de combate à violência contra a pessoa idosa, verificou-se que todas as regiões registram poucos casos de internação por maus-tratos, como pelo menos uma das causas, com notoriedade para o Norte e Centro-Oeste, que por diversos anos não registraram nenhum caso. As regiões Sul e Sudeste apresentaram maior número de internações por maus-tratos em relação às demais, no entanto isso pode ter ocorrido devido à grande subnotificação de casos, o que intensifica ainda mais a gravidade do problema de indicadores sobre esse tema ${ }^{(3)}$.

Em síntese, os indicadores de saúde para a política de saúde da pessoa idosa relacionados ao acesso aos serviços de saúde, assistência social, alimentação adequada, consumo abusivo e frequente de álcool, fisicamente inativos, fumantes, moradia adequada, participação social, prevenção e manutenção da capacidade funcional e violência e maus-tratos são corroborados por outros autores ${ }^{(17,24-30)}$.

Reconhece-se, que apesar de ser possível calcular indicadores para o acompanhamento de políticas a partir das fontes de dados disponíveis, limitações concernentes à oportunidade dos dados, sua desagregabilidade e confiabilidade restringem sua utilização para o aprimoramento das políticas nacionais ${ }^{(3)}$. Validade, confiabilidade, sensibilidade e especificidade, transparência metodológica, comunicabilidade, periodicidade de atualização do 
indicador, e comparabilidade ao longo do tempo são características fundamentais para elaboração de um bom indicador ${ }^{(22,23)}$.

\section{Indicadores relacionados ao serviço de farmácia}

Ao avaliar o grau de conhecimento de utentes sobre o tratamento farmacológico realizado e sua satisfação em frequentar do Centro de Atenção Especializado ao Idoso (CAEI), $79 \%(\mathrm{n}=79)$ auto-referiram se sentir bem informado sobre os medicamentos utilizados e $96 \%$ $(\mathrm{n}=96)$ registraram ser importante estar bem informado sobre o seu tratamento (Tabela 2).

Tabela 02 - Grau de conhecimento e satisfação dos utentes

\begin{tabular}{|c|c|c|}
\hline PARÂMETROS & Sim & Não \\
\hline $\begin{array}{l}\text { O utente conhece/sabe para que serve(m) o(s) medicamento(s) que irá utilizar e/ou } \\
\text { utiliza? }\end{array}$ & $91(91 \%)$ & $9(9 \%)$ \\
\hline O utente conhece/sabe as dose(s) que deve tomar do(s) medicamento(s)? & $90(90 \%)$ & $10(10 \%)$ \\
\hline O utente conhece/sabe os horários de administração dos medicamentos & $97(97 \%)$ & $3(3 \%)$ \\
\hline O utente sabe o modo de tomar corretamente os medicamentos? & $88(88 \%)$ & $12(12 \%)$ \\
\hline O utente usa e/ou toma o medicamento sem ajuda de ninguém? & $80(80 \%)$ & $20(20 \%)$ \\
\hline O utente precisa de alguém para lhe administrar o medicamento? & $16(16 \%)$ & $84(84 \%)$ \\
\hline O utente sabe as restrições durante o uso dos medicamentos? & $21(21 \%)$ & $79(79 \%)$ \\
\hline O utente sabe dizer se os medicamentos podem causar alguma reação adversa? & $23(23 \%)$ & $77(77 \%)$ \\
\hline O utente sabe dizer se os medicamentos podem causar algum evento adverso? & $23(23 \%)$ & $77(77 \%)$ \\
\hline O utente sabe o que deve fazer quando esquecer de tomar a medicação? & $43(43 \%)$ & $57(57 \%)$ \\
\hline $\begin{array}{l}\text { O utente sabe se sobre potenciais interações entre alimentos-medicamentos e } \\
\text { medicamentos-medicamentos }\end{array}$ & $11(11 \%)$ & $89(89 \%)$ \\
\hline $\begin{array}{l}\text { O utente sabe se os medicamentos que usa podem interferir em exames } \\
\text { laboratoriais? }\end{array}$ & $11(11 \%)$ & $89(89 \%)$ \\
\hline O utente se sente bem informado sobre a medicação a ser utilizada? & $79(79 \%)$ & $21(21 \%)$ \\
\hline O utente acredita ser importante estar bem informado sobre o seu tratamento? & $96(96 \%)$ & $4(4 \%)$ \\
\hline O utente se sente respeitado o Casa do Idoso? & $98(98 \%)$ & $2(2 \%)$ \\
\hline O utente se sente bem ao frequentar o Casa do Idoso? & $99(99 \%)$ & $1(1 \%)$ \\
\hline O utente gosta de frequentar o Casa do Idoso? & $97(97 \%)$ & $3(3 \%)$ \\
\hline
\end{tabular}

Fonte: Indicadores de saúde e de assistência farmacêutica voltadas à população idosa no Centro de Atenção Especializada do idoso de Belém, 2020.

$79 \%(\mathrm{n}=79)$ auto-referiram não saber sobre as restrições existentes durante o uso de medicamentos. $89 \%(n=89)$ auto-referiram não saber sobre potenciais interações entre alimentos-medicamentos, medicamentos-medicamentos e de interferência que os medicamentos podem causar em exames laboratoriais. $77 \%(\mathrm{n}=77)$ auto-referiram não saber sobre o medicamento poder causar reações adversas. 57\% $(\mathrm{n}=57)$ auto-referiram não saber sobre o que fazer quando esquecia de tomar os medicamentos, informando que tomavam assim que lembravam e/ou que tomavam o dobro da dose para suprir a dose esquecida (Tabela 2). 
98\% ( $\mathrm{n}=98)$ auto-referiram se sentir respeitado no Centro de Atenção Especializado ao Idoso $(\mathrm{CAEI}), 99 \%(\mathrm{n}=99)$ se sentia bem ao frequentar o CAEI e $97 \%(\mathrm{n}=97)$ gostava de frequentar o Casa do Idoso. O grau de conhecimento e de satisfação dos utentes foi classificado como médio $55 \%$ ( $\mathrm{n}=55$ ), seguindo Escala Likert; ${ }^{(19)}$ um tipo de escala de resposta psicométrica usada habitualmente em questionários, sendo a escala mais usada em pesquisas de opinião. Pode-se, aqui inferir que esse grau de satisfação seja um resultado do processo de acolhimento e do perfil profissional da equipe multidisciplinar.

Quanto ao perfil de prescrições, tendo como base os critérios regulamentados segundo a legislação vigente, $49 \%(\mathrm{n}=49)$ das prescrições estavam em desacordo. O nome dos utentes foram encontrados em 100\% $(n=100)$ das prescrições. Peso e endereço não estavam registrados em 100\% ( $n=100$. Em síntese, a ausência de informação interfere na comunicação interpessoal dos profissionais, refletindo em erros de medicação (Tabela 3). Assim, de acordo com Escala Likert; ${ }^{(19)}$ a classificação do perfil de prescrições e o nível de prescrição foi avaliado como bom, tendo uma média de $63 \%(\mathrm{n}=63)$.

Foi observado potenciais interações de medicamentos. $\mathrm{O}$ primeiro trimestre $\left(1^{\circ} \mathrm{T}\right)$ apresentou um total de 113 interações (Média: 37,7; Desvio Padrão: 28,7); Segundo trimestre $\left(2^{\circ} \mathrm{T}\right) 129$ (Média: 43 e Desvio Padrão: 31,4); Terceiro trimestre $\left(3^{\circ} \mathrm{T}\right) 133$ (Média: 44,3 e Desvio Padrão: 29,7); e Quarto trimestre (4º 136 (Média: 45,3 e Desvio Padrão: 29,5); ou seja, percebe-se um crescimento quanto ao número de interações ao longo do ano (Tabela 4).

Foram encontrados 27 medicamentos (fármacos) que se enquadram nos critérios de Beers-Fick, ${ }^{(12)}$ representando 13,8\% dos medicamentos padronizados pelo Centro de Atenção Especializado ao Idoso (CAEI) ${ }^{(17)}$. Outrossim, entre estes, encontra-se o omeprazol, que não deve ser utilizado por mais que 8 (oito) semanas seguidas. Pelo fato das prescrições não registrarem duração do tratamento, observou-se que o omeprazol se tornou de uso contínuo, dispensado para três meses; visto que, as consultas são realizadas por trimestres.

Quanto a legibilidade das prescrições, 51\% $(\mathrm{n}=51)$ estavam de acordo com a legislação vigente (Tabela 3). No Brasil, para prescrições não eletrônicas, a prescrição de medicamentos é normatizada pela Lei $\mathrm{n}^{\circ}$ 5.991, de 17 de dezembro de 1973, pela Lei $\mathrm{n}^{\circ}$ 9.787, de 10 de fevereiro de 1999 e pela Resolução do Conselho Federal de Farmácia n 357, de 27 de abril de 2001. Destaca-se que a receita deve ser escrita à tinta, em letra de forma, clara e por extenso, como especificado na legislação ${ }^{(33)}$.

Quanto aos medicamentos potencialmente inapropriados para os idosos, foram encontrados 13 fármacos que se enquadram nos critérios de Beers-Fick e que estão 
padronizados no Centro de Atenção Especializado ao Idoso (CAEI), representando 13,8\% dos medicamentos padronizados. Este fato já havia sido identificado e informado por Pereira em $2018^{(17)}$. Os medicamentos identificados foram: ácido acetil salicílico, alprazolam, amitriptilina, bronfeniranamina, cetoprofeno, ciclobenzaprina, clonazepam, diazepam, enalapril, escitalopram, esomeprazol, espironolactona, fenobarbital, fluoxetina, ibuprofeno, insulina, levotiroxina T4, meloxicam, nifedipino, nortriptilina, óleo mineral, omeprazol, pantoprazol, prometazina, ranitidina, sertralina e zolpidem.

Tabela 03 - Perfil de prescrições no Centro de Atenção Especializado ao Idoso

\begin{tabular}{lcc}
\hline \multicolumn{1}{c}{ Dados da Prescrição } & De acordo com a legislação & Em desacordo com a legislação \\
\hline Legibilidade & $51(51 \%)$ & $49(49 \%)$ \\
Escrita por extenso & $73(73 \%)$ & $27(27 \%$ \\
Denominação genérica & $77(77 \%)$ & $23(23 \%)$ \\
Forma farmacêutica & $96(96 \%)$ & $4(4 \%)$ \\
Dosagem & $98(98 \%)$ & $2(2 \%)$ \\
Técnica de administração & $60(60 \%)$ & $40(40 \%)$ \\
Horário de administração & $45(45 \%)$ & $55(55 \%)$ \\
Via de administração & $83(83 \%)$ & $17(17 \%)$ \\
Duração do tratamento & $39(39 \%)$ & $61(61 \%)$ \\
Nome do utente & $100(100 \%)$ & 0 \\
Peso do utente & 0 & $100(100 \%)$ \\
Endereço de utente & 0 & $100(100 \%)$ \\
Endereço da instituição & $97(97 \%)$ & $3(3 \%)$ \\
Assinatura do médico & $94(94 \%)$ & $6(6 \%)$ \\
Carimbo do médico & $96(96 \%)$ & $4(4 \%)$ \\
CRM & $96(96 \%)$ & $4(4 \%)$ \\
Data & $100(100 \%)$ & 0 \\
\hline
\end{tabular}

Fonte: Indicadores de saúde e de assistência farmacêutica voltadas à população idosa no Centro de Atenção Especializada do idoso de Belém, 2020.

De acordo com Beers-Fick, seus critérios vem sendo aplicados para o monitoramento de prescrições e utilização de medicamentos em utentes não institucionalizados, hoje incorpora também, critérios adicionais para o uso de medicamentos em idosos com patologias específicas, resultando em uma lista com 28 medicamentos ou classes de medicamentos inadequados e 35 medicamentos ou classes de medicamentos inadequados em 15 patologias especificas ${ }^{(12,17)}$. Desde 1991 a Lista de Beers-Fick ${ }^{(12)}$ vem sendo empregada para avaliar a prescrição de medicamentos potencialmente inapropriados para idosos ${ }^{(17,20)}$.

Em adição, foram encontradas potenciais interações de medicamentos em 92 prescrições (Quadro 1). Os medicamentos comumente envolvidos são aqueles usados no manejo cotidiano 
do utente idoso com doenças crônicas. Digoxina, diuréticos, hipoglicemiantes, antiarrítmicos, varfarina, antiinflamatórios não-esteroidais, fenitoína, analgésicos de ação central e antipsicóticos aparecem frequentemente nas listas de potenciais interações medicamentosas, maioria dos medicamentos prescritos na instituição ${ }^{(12,17,21)}$. Os medicamentos comumente envolvidos são aqueles usados no cotidiano do manejo do utente idoso com doenças crônicas. Digoxina, diuréticos, hipoglicemiantes, antiarrítmicos, varfarina, antiinflamatórios nãoesteroidais, fenitoína, analgésicos de ação central e antipsicóticos aparecem frequentemente nas listas de potenciais interações medicamentosas, maioria dos medicamentos prescritos na instituição.

Tabela 4 - Potenciais interações de medicamentos prescritos nos quatro trimestres de 2019

\begin{tabular}{lcccc}
\hline \multicolumn{1}{c}{ Grau de Interação } & $\mathbf{1}^{\mathbf{o}} \mathbf{T}$ & $\mathbf{2}^{\mathbf{o}} \mathbf{T}$ & $\mathbf{3}^{\mathbf{o}} \mathbf{T}$ & $\mathbf{4}^{\mathbf{0}} \mathbf{T}$ \\
\hline Sérias (Usar alternativas) & 15 & 21 & 22 & 24 \\
Moderadas (Monitorar de perto) & 70 & 79 & 78 & 79 \\
Leves (Menores) & 28 & 29 & 33 & 33 \\
Total & 113 & 129 & 133 & 136 \\
Média & 37,7 & 43 & 44,3 & 45,3 \\
Desvio Padrão & 28,7 & 31,4 & 29,7 & 29,5 \\
\hline
\end{tabular}

Fonte: Indicadores de saúde e de assistência farmacêutica voltadas à população idosa no Centro de Atenção Especializada do idoso de Belém,2020.

Importante, também, foram os 113 potenciais interações de medicamentos observadas no primeiro trimestre, 129 no segundo trimestre, 133 no terceiro trimestre e 136 no quarto trimestre; ou seja, observa-se um leve crescimento quanto ao número de interações ao longo do ano (Tabela 4). A gravidade e as possíveis consequências das interações de medicamentos estão relacionadas às condições clínicas dos indivíduos, a quantidade e as características dos medicamentos. Estima-se que o risco de apresentar interação medicamentosa aumenta de $13 \%$ com o uso de dois medicamentos para $58 \%$ com o uso de cinco medicamentos, e, ainda com o uso de sete ou mais medicamentos a incidência eleva-se para $82 \%$, denotando graves riscos ao utente $^{(21,32)}$.

O Quadro 1 (Potenciais interações de medicamentos em uso pelos utentes do Centro de Atenção Especializado ao Idoso) três categorias de interações medicamentosas: Interação medicamentosa séria: necessidade de usar medicamentos alternativos; Interação medicamentosa moderada: necessidade de monitorar de perto; Interação medicamentosa leve: menor risco. Sabe-se, que durante o tratamento farmacológico de idosos, é comum encontrar prescrições com potenciais interações medicamentosas. Interações medicamentosas são uma 
resposta farmacológica ou clínica à administração de uma combinação de medicamentos, que difere do esperado para cada um dos agentes dados isoladamente ${ }^{(21)}$.

As principais situações de risco para interações incluem a polimedicação, o utente que recebe cuidados de vários médicos e a automedicação ${ }^{(35)}$. Estudos corroboram que a polifarmácia, prescrição de medicamentos contraindicados e o uso de dois ou mais fármacos com a mesma atividade farmacológica favorecem potenciais interações de medicamentos, eventos adversos e reações adversas ${ }^{(31-35)}$. Outrossim, o uso de medicamentos concomitantes comprometem, de forma significativa, resultados de exames laboratoriais, colaborando para o diagnóstico falso-positivo ou falso-negativo ${ }^{(25,31)}$. Em tempo, é comum quando os idosos esquecem de tomar os medicamentos, tomarem assim que se lembram ou tomarem o dobro da dose no horário subsequente para suprir a dose esquecida ${ }^{(34,35)}$.

Quanto ao perfil de dispensação de medicamentos, observou-se que possuem grau médio de conhecimento sobre a farmacoterapia estabelecida para os utentes idosos, utilizandose Escala Likert ${ }^{(19)}$. Observou-se que as profissionais não pactuam os objetivos terapêuticos, não selecionam estratégias terapêuticas, não fazem advertências sobre os possíveis efeitos adversos e sobre interferências entre medicamentos e diagnósticos laboratoriais (Tabela 5).

Quanto ao perfil de dispensação de medicamentos, observou-se por parte dos farmacêuticos, um grau médio de conhecimento sobre as estratégias farmacoterapêuticas. Os indicadores avaliados permitem inferir que o cuidado farmacêutico ainda não foi implantado (Tabela 5). O cuidado farmacêutico constitui a ação integrada do farmacêutico com a equipe de saúde, centrada no usuário, para promoção, proteção e recuperação da saúde e prevenção de agravos. Visa à educação em saúde e à promoção do uso racional de medicamentos prescritos e não prescritos, de terapias alternativas e complementares, por meio dos serviços da clínica farmacêutica e das atividades técnico-pedagógicas voltadas ao indivíduo, à família, à comunidade e à equipe de saúde ${ }^{(36)}$.

Os profissionais farmacêuticos justificaram que o cuidado farmacêutico não foi ainda implantado em função da estrutura física inadequada da farmácia e do tempo; visto que, além da dispensação é preciso realizar atividades operacionais da logística da assistência farmacêutica. Entretanto, as atividades que podem ser desenvolvidas e que não demandam muito tempo são desempenhadas; tais como, esclarecer ao utente sobre os medicamentos selecionados, sobre as formas farmacêuticas e os esquemas de administração, quais os efeitos terapêuticos esperados e checagem sobre a compreensão do utente sobre o esquema terapêutico estabelecido. Esses achados estão em concordância com outros estudos que afirmam que a 
orientação aos utentes se torna praticamente inviável em decorrência da estrutura e organização da gestão técnica da assistência e da gestão clínica do medicamento ${ }^{(13,17,36)}$.

Quadro 1 - Potenciais interações de medicamentos em uso pelos utentes do Centro de Atenção Especializado ao Idoso

\begin{tabular}{|c|c|}
\hline \multicolumn{2}{|c|}{ INTERAÇÃO MEDICAMENTOSA SÉRIA: Necessidade de usar medicamentos alternativos } \\
\hline AAS + captopril & Antagonismo farmacodinâmico. \\
\hline AAS + enalapril & Antagonismo farmacodinâmico. \\
\hline Amiodarona + sinvastatina & A amiodarona aumenta a toxicidade da sinvastatina, diminuindo o metabolismo. \\
\hline Anlodipino + sinvastatina & Anlodipino aumenta os níveis de sinvastatina. \\
\hline Anlodipino + sinvastatina & Anlodipino aumenta os níveis de sinvastatina. \\
\hline Carbamazepina + sinvastatina & $\begin{array}{l}\text { A carbamazepina diminuirá o nível ou o efeito da sinvastatina, afetando o } \\
\text { metabolismo da enzima hepática CYP3A4 }\end{array}$ \\
\hline Escitalopram + trazodona & Escitalopram e trazodona aumentam os níveis de serotonina. \\
\hline Fluconazol + sinvastatina & $\begin{array}{l}\text { O fluconazol aumentará o nível ou o efeito da sinvastatina, afetando o metabolismo } \\
\text { da enzima hepática CYP3A4. }\end{array}$ \\
\hline Ibuprofeno + AAS & O ibuprofeno aumenta a toxicidade da aspirina por anticoagulação \\
\hline Ibuprofeno + enalapril & Antagonismo farmacodinâmico. \\
\hline Losartana + enalapril & Aumenta a toxicidade do outro pelo sinergismo farmacodinâmico. \\
\hline Nifedipino + sinvastatina & $\begin{array}{l}\text { A nifedipino aumentará o nível ou o efeito da sinvastatina, afetando o metabolismo } \\
\text { da enzima hepática CYP3A4. }\end{array}$ \\
\hline Prednisona + sinvastatina & $\begin{array}{l}\text { A prednisona diminuirá o nível ou o efeito da sinvastatina, afetando o metabolismo } \\
\text { da enzima hepática CYP3A4 }\end{array}$ \\
\hline \multicolumn{2}{|c|}{ INTERAÇÃO MEDICAMENTOSA MODERADA: Necessidade de monitorar de perto } \\
\hline AAS + insulina NPH & A aspirina aumenta os efeitos da insulina NPH pelo sinergismo farmacodinâmico. \\
\hline Amiodarona + losartana & A amiodarona diminui os efeitos do losartana diminuindo o metabolismo. \\
\hline Atenolol + valsartana & Sinergismo farmacodinâmico. \\
\hline Carbamazepina + losartana & $\begin{array}{l}\text { A carbamazepina diminui o nível ou o efeito do losartana, afetando o metabolismo } \\
\text { da enzima hepática CYP2C9. }\end{array}$ \\
\hline Espironolactona + hidroclorotiazida & A espironolactona aumenta e a hidroclorotiazida diminui o potássio sérico. \\
\hline Losartana + diclofenaco & Aumenta a toxicidade um do outro. \\
\hline Losartana + espironolactona & Aumentam o potássio sérico. \\
\hline Losartana + furosemida & Losartana aumenta e a furosemida diminui o potássio sérico. \\
\hline Losartana + hidroclorotiazida & Losartana aumenta e a hidroclorotiazida diminui o potássio sérico \\
\hline Losartana + insulina NPH & $\begin{array}{l}\text { Losartana aumenta os efeitos da insulina NPH por mecanismo de interação não } \\
\text { especificado. }\end{array}$ \\
\hline Omeprazol + ciprofloxacino & $\begin{array}{l}\text { O omeprazol diminuirá o nível ou o efeito da ciprofloxacino por mecanismo } \\
\text { desconhecido }\end{array}$ \\
\hline Omeprazol + losartana & $\begin{array}{l}\text { O omeprazol aumentará o nível ou o efeito do losartana, afetando o metabolismo da } \\
\text { enzima hepática CYP2C9. }\end{array}$ \\
\hline Sinvastatina + valsartana & A sinvastatina aumentará o nível ou o efeito do valsartana. \\
\hline Valsartana + espironolactona & Valsartana e espironolactona aumentam o potássio sérico. \\
\hline \multicolumn{2}{|r|}{ INTERAÇÃO MEDICAMENTOSA LEVE: menor risco } \\
\hline Diclofenaco + meloxicam & $\begin{array}{l}\text { O diclofenaco aumentará o nível ou o efeito do meloxicam pela competição ácida } \\
\text { (aniônica) de medicamentos pela depuração tubular renal. }\end{array}$ \\
\hline Furosemida + sertralina & Sinergismo farmacodinâmico. \\
\hline Hidroclorotiazida + AAS & $\begin{array}{l}\text { A hidroclorotiazida aumentará o nível ou o efeito da aspirina pela competição ácida } \\
\text { (aniônica) por fármaco pela depuração tubular renal. }\end{array}$ \\
\hline Hidroclorotiazida + cetoprofeno & $\begin{array}{l}\text { A hidroclorotiazida aumentará o nível ou o efeito do cetoprofeno pela competição } \\
\text { ácida (aniônica) com medicamentos pela depuração tubular renal. }\end{array}$ \\
\hline $\begin{array}{l}\text { Hidroclorotiazida + hidróxido de } \\
\text { magnésio }\end{array}$ & $\begin{array}{l}\text { A hidroclorotiazida diminui os níveis de hidróxido de magnésio aumentando a } \\
\text { depuração renal. }\end{array}$ \\
\hline Hidroclorotiazida + ibuprofeno & $\begin{array}{l}\text { A hidroclorotiazida aumentará o nível ou o efeito do ibuprofeno pela competição } \\
\text { ácida (aniônica) com medicamentos pela depuração tubular renal. }\end{array}$ \\
\hline Hidroclorotiazida + insulina NPH & $\begin{array}{l}\text { A hidroclorotiazida diminui os efeitos da insulina NPH por antagonismo } \\
\text { farmacodinâmico. }\end{array}$ \\
\hline Hidroclorotiazida + metformina & $\begin{array}{l}\text { A hidroclorotiazida diminui os efeitos da metformina pelo antagonismo } \\
\text { farmacodinâmico. }\end{array}$ \\
\hline Nifedipino + metformina & O nifedipino aumenta os níveis de metformina aumentando a absorção GI. \\
\hline Prednisona + hidroclorotiazida & Sinergismo farmacodinâmico. \\
\hline Sinvastatina + loratadina & $\begin{array}{l}\text { A sinvastatina aumentará o nível ou o efeito da loratadina pelo transportador de } \\
\text { efluxo de glicoproteína P (MDR1). }\end{array}$ \\
\hline
\end{tabular}


Fonte: Indicadores de saúde e de assistência farmacêutica voltadas à população idosa no Centro de Atenção Especializada do idoso de Belém, 2020.

Tabela 5 - Perfil de dispensação de medicamentos no Centro de Atenção Especializado ao Idoso

\begin{tabular}{lcc}
\hline \multicolumn{1}{c}{ PARÁMETROS } & SIM & NÃO \\
\hline Dialoga com o utente sobre o problema de saúde? & $100 \%$ & $0 \%$ \\
Pactua os objetivos terapêuticos com o utente? & $0 \%$ & $100 \%$ \\
Esclarece as estratégias terapêuticas? & $0 \%$ & $100 \%$ \\
Esclarece medidas não-farmacológicas? & $50 \%$ & $50 \%$ \\
Esclarece ao utente sobre o(s) medicamento(s) selecionado(s)? & $100 \%$ & $0 \%$ \\
Esclarece ao utente sobre a(s) forma(s) farmacêutica(s) e o(s) esquema(s) de administração? & $100 \%$ & $0 \%$ \\
Explicita ao utente sobre os efeitos terapêuticos esperados? & $100 \%$ & $0 \%$ \\
Faz advertências sobre os possíveis efeitos adversos? & $0 \%$ & $100 \%$ \\
Faz advertências sobre interações com alimentos e outros medicamentos? & $50 \%$ & $50 \%$ \\
Faz advertências sobre interferências entre o(s) medicamento(s) e diagnósticos laboratoriais? & $0 \%$ & $100 \%$ \\
Faz checagem acerca da compreensão do utente sobre o esquema terapêutico estabelecido? & $100 \%$ & $0 \%$ \\
Agenda nova consulta para o monitoramento do tratamento com o utente? & $0 \%$ & $100 \%$ \\
Preocupa-se com a adesão ao tratamento pelo utente? & $100 \%$ & $0 \%$ \\
\hline
\end{tabular}

Fonte: Indicadores de saúde e de assistência farmacêutica voltadas à população idosa no Centro de Atenção Especializada do idoso de Belém, 2020.

\section{Viés e limitação}

Registra-se a possibilidade de viés quanto a forma de recrutamento; ou seja, amostragem de conveniência. O número de indivíduos participantes é pequeno; quiçá por se tratar de um grupo de idosos com comorbidades delimitadas, o que dificulta a generalização dos achados. Quanto as limitações, houve dificuldade de acesso a dados e informações complementares; bem como, deparou-se com prontuários incompletos e desatualizados.

\section{CONCLUSÃO}

Os achados relacionados a indicadores de determinantes sociais, equidade e política de saúde da pessoa idosa permitem inferir que os usuários do Centro de Atenção Especializado ao Idoso seguem o perfil brasileiro. Os indicadores de perfil de prescrições, critérios de BeersFick, interações de medicamentos, dispensação farmacêutica permitem inferir a necessidade do aprimoramento do serviço farmacêutico.

\section{REFERÊNCIAS BIBLIOGRÁFICAS}

1. Ministério da Saúde (BR). Secretaria de Atenção à Saúde. Diretrizes para o cuidado das 
pessoas idosas no SUS: proposta de modelo de atenção integral. Brasília, DF, 2014. Site: www.saude.gov.br/idoso

2. Bezerra FC, Almeida MI, Nóbrega-Therrien SM. Estudos sobre envelhecimento no Brasil: revisão bibliográfica. Rev. Bras. Geriatr. Gerontol. Rio de Janeiro, 2012; 15(1):155-167. Online version ISSN 1981-2256

3. Romero DE, Pires DC, Marques A, Muzy J. Diretrizes e indicadores de acompanhamento das políticas de proteção à saúde da pessoa idosa no Brasil. Reciis - Rev Eletron Comun Inf Inov Saúde. $\quad 2019 ; 13(1): 134-157 . \quad$ ISSN $\quad$ 1981-6278. DOI: https://doi.org/10.29397/reciis.v13i1.1569

4. Congresso nacional (BR). Lei $\mathrm{n}^{\circ}$ 8.080, de 19 de outubro de 1990. Dispõe sobre as condições para a promoção, proteção e recuperação da saúde, a organização e o funcionamento dos serviços correspondentes e dá outras providências. Brasília. DF.

5. Congresso nacional (BR). Lei ${ }^{\circ}$ 8.842, de 4 de janeiro de 1994. Regulamenta a política nacional do idoso, cria o Conselho Nacional do Idoso e dá outras providências. Brasília. DF.

6. Congresso nacional (BR). Lei $\mathrm{n}^{\mathrm{o}} 10741$, de 1 de outubro de 2003. Dispõe sobre os direitos do idoso à vida, à liberdade, ao respeito, à dignidade, aos alimentos, à saúde, à convivência familiar e comunitária, entre outros direitos fundamentais. Brasília. DF, 2003.

7. Ministério da Saúde (BR). Ministério da Saúde. Portaria MS/GM no 2528, de outubro de 2006. Dispõe sobre medidas coletivas e individuais de saúde para a população idosa em consonância com os princípios e diretrizes do Sistema Único de Saúde, a fim de promover a recuperação, a autonomia e a independência dos idosos. Brasília. DF, 2006.

8. Nascimento RCRM, Álvares J, Junior AAG, Gomes, IC, Silveira MR, Costa EA, Leite SN, Costa KS, Soeiro OM, Guibu IA, Karnikowski MGO, Acurcio FA. Polifarmácia: uma realidade na atenção primária do Sistema Único de Saúde. Revista de Saúde Pública. 2017;51(2):1-12. DOI: $10.11606 / \mathrm{S} 1518-8787.2017051007136$

9. Veras RP, Gomes JAC, Macedo ST. A coordenação de cuidados amplia a qualidade assistencial e reduz custos. Rev. bras. geriatr. gerontol. [Internet]. 2019; 22(2):e190073. https://doi.org/10.1590/1981-22562019022.190073. ISBN 1981225620190

10. Davidoff AJ, Miller GE, Sarpong EM, Yang E, Brandt N, Fick DM. Prevalence of potentially inappropriate medication use in older adults using the 2012 Beers criteria. J Am Geriatr Soc. 2015;63(3):486-500. DOI: 10.1111/jgs.13320.

11. Carvalho BAF, Santos MAP, Nogueira FRS, Sá GGM, Oliveira-Neto JG, Martins MCC, Santos EP. Prevalência da prática de exercícios físicos em idosos e sua relação com as dificuldades e a falta de aconselhamento profissional específico. R. bras. Ci. e Mov, 2017;25(1):29-40. ISSN: 0103-1716.

12. Fick DM. American Geriatrics Society 2015 Beers Criteria Update Expert Panel.American. Geriatrics Society Updated Beers Criteria for Potentially Inappropriate Medication Use in Older Adults. J Am Geriatr Soc, 2015;63(11):2227-46. DOI: 10.1111/jgs.13702

13. Soler O, Barreto JOM. Community-Level Pharmaceutical Interventions to Reduce the Risks 
of Polypharmacy in the Elderly: Overview of Systematic Reviews and Economic Evaluations. Frontiers in Pharmacology. 2019;10:e10:302. DOI: 10.3389/fphar.2019.00302.

14. Minayo MCS. Análise qualitativa: teoria, passos e fidedignidade. Ciência \& Saúde Coletiva, 2012;17(3):621-626, DOI: 10.1590/S1413-81232012000300007

15. Yin RK. Estudo de caso: planejamento e métodos. 5. ed. Bookman: Porto Alegre, RS, Brasil. 2015. ISBN 978-85-8260-232-4

16. O’neill J, Tabish H, Welch V, Petticrew M, Pottie K, Clarke M, Evans T, Pardo JP, Waters E, White H, Tugwell P. Applying an equity lens to interventions: using PROGRESS ensures consideration of socially stratifying factors to illuminate inequities in health. J Clin Epidemiol (2014) 67:56-64. DOI: 10.1016/j.jclinepi.2013.08.005

17. Pereira LAR. Perfil de prescrições médicas de idosos utentes do centro de atenção especializada do idoso de Belém à luz dos Critérios de Beers-Fick. Belém. Monografia (Trabalho de Conclusão de Curso). 2018. Universidade Federal do Pará: Faculdade de Farmácia. Pará.

18. Likert R. A Technique for the Measurement of Attitudes. Arch Psychol. 1932; 140: 1-55. ISSN 2169-3269.

19. Souza JM, Thomson JC, Catisti DG. Avaliação de prescrições medicamentosas de um hospital universitário brasileiro. Rev. bras. educ. med. [online]. 2008;32(2):188-196. ISSN 1981-5271. http://dx.doi.org/10.1590/S0100-55022008000200006.

20. Pinto JM, Neri AL. Trajetórias da participação social na velhice: uma revisão sistemática da literatura. Rev. bras. geriatr. gerontol. 2017;20(2):259-272. https://doi.org/10.1590/198122562017020.160077 .

21. Souza DM, Souza LB, Lana GG, Souza SM, Aguilar NC, Silva DR. Uso Inapropriado de Medicamentos pelo Idoso: Polifarmácia e seus Efeitos. Pensar Acadêmico. 2018;16(2):166178. ISSN 1808-6136.

22. Jannuzzi PM. Indicadores para diagnóstico, monitoramento e avaliação de programas sociais no Brasil. Revista do Serviço Público. 2005;56(2):137-160. DOI: 10.21874/rsp.v56i2.222

23. Melo DO; Silva SRA, Castro LLC. Avaliação de indicadores de qualidade de prescrição de medicamentos em uma unidade de atenção primária com diferentes modelos de atenção. Epidemiologia e Serviços de Saúde. 2016;25:259-270. DOI: 10.5123/S167949742016000200005

24. Obreli-Neto PR, Marusic S, Guidoni CM, Baldoni AO, Renovato RD, Pilger D, Cuman RKN, Pereira LRL. Economic evaluation of a pharmaceutical care program for elderly diabetic and hypertensive patients in primary health care: a 36-month randomized controlled clinical trial. Journal of managed care \& specialty pharmacy. 2015;21(1):66-75. ISSN 10834087. DOI: 10.18553 / jmcp.2015.21.1.66

25. Oliveira PC. Prevalência e Fatores Associados à Polifarmácia entre Idosos Atendidos na Atenção Primária a Saúde em Belo Horizonte. Mestrado (Dissertação). 2018. Universidade 
Federal de Minas: Mestrado em Medicamentos e Assistência Farmacêutica. Minas Gerais.

26. Matos ASB, Chaves EC, Gonçalves LHT. Serviços de proteção básica e especial de assistência social aos idosos no município de Belém, PA. Revista Kairós: Gerontologia. 2018;21(2):253-266. DOI: 10.23925/2176-901X.2018v21i2p253-266

27. Miguel EN. O morar contemporâneo do idoso: Onde e como reside no estado de Minas Gerais. Viçosa. Mestrado (Dissertação). 2016. Universidade Federal de Viçosa: Mestrado em Economia Doméstica. Minas Gerais.

28. Miranda RNA, Carvalho EP, Amorim YR, Santos KS, Serrão FO. Conhecendo a saúde Nutricional de idosos atendidos em uma Organização não Governamental, Benevides/PA. Revista Conexão UEPG. 2017;13(3):512-529. DOI: 10.5212/rev.conexao.v.13.i3.0013

29. Santos MD, Silva MF, Velloza LA, Pompeu JE. Falta de acessibilidade no transporte público e inadequação de calçadas: efeitos na participação social de pessoas idosas com limitações funcionais. Revista Brasileira de Geriatria e Gerontologia. 2017;20(2):161-174. DOI: 10.4090/juee.2008.v2n2.033040

30. Sobreira FMM, Sarmento WE, Oliveira AMB. Perfil epidemiológico e sociodemográfico de idosos frequentadores de grupo de convivência e satisfação quanto à participação no mesmo. Revista Brasileira de Ciencias da Saude. 2011;15(4):429-438. DOI: 10.4034/RBCS.2011.15.04.08

31. Souza AS, Santiago EC, Almeida LC. Interferências nos exames laboratoriais causados pelo anti-hipertensivos usados no brasil. Revista Eletrônica Atualiza Saúde. 2016;3(3):101-113. ISSN: 2359-4470.

32. Locatelli J. Interações medicamentosas em idosos hospitalizados. Einstein (São Paulo). 2007;5(4):343-346. ISSN 1679-4508.

33. Madruga CM.D, Souza ESM. Manual de orientações básicas para prescrição médica $-2^{\mathrm{a}}$ ed. rev. ampl. Brasília: 2011. https://portal.cfm.org.br/images/stories/biblioteca/cartilhaprescrimed2012.pdf

34. Martins FP, Maia HU, Pereira LSM. Desempenho de idosos em testes funcionais e o uso de medicamentos. Fisioterapia em Movimento (Curitiba). 2007;20(1): 85-92. ISSN 01035150

35. Montoya IC, Sánchez-Castellano C, Cruz-Jentoft AJ. Mejorando la prescripción de medicamentos en las personas mayores: una nueva edición de los criterios STOPP-START. Rev Esp Geriatr Gerontol, 2015;50:89-96 - DOI: 10.1016/j.regg.2014.10.005

35. Ministério da Saúde (BR). Secretaria de Ciência, Tecnologia e Insumos Estratégicos. Departamento de Assistência Farmacêutica e Insumos Estratégicos. Serviços farmacêuticos na atenção básica à saúde / Ministério da Saúde, Secretaria de Ciência, Tecnologia e Insumos Estratégicos. Departamento de Assistência Farmacêutica e Insumos Estratégicos. - Brasília : Ministério da Saúde, 2014. 108 p. : il. - (Cuidado farmacêutico na atenção básica ; caderno 1). ISBN 978-85-334-2196-7 